Case Report

\title{
Uterine Rupture Secondary to Placenta Percreta on Previa: A Case Report of Successful Management by Caesarian Hysterectomy
}

\author{
Dawit Sereke ${ }^{1,}$, , Habte Hailemelekot ${ }^{1}$, Abduselam Hagos ${ }^{2}$, Elias Teages Adgoy ${ }^{3}$ \\ ${ }^{1}$ Department of Obstetrics and Gynecology, Mendefera Regional Referral Hospital, Mendefera, Eritrea \\ ${ }^{2}$ Department of Obstetrics and Gynecology, Orotta National Referral Hospital, Asmara, Eritrea \\ ${ }^{3}$ Department of Community Medicine and Primary Health Care, Orotta School of Medicine and Dentistry, Asmara, Eritrea \\ Email address: \\ weldegebrealdawit@gmail.com (D. Sereke),Abduselam1084@gmail.com(A. Hagos), eliasteages@gmail.com (E. T. Adgoy) \\ ${ }^{*}$ Corresponding author
}

\section{To cite this article:}

Dawit Sereke, Habte Hailemelekot, Abduselam Hagos, Elias Teages Adgoy. Uterine Rupture Secondary to Placenta Percreta on Previa: A Case Report of Successful Management by Caesarian Hysterectomy. Science Journal of Public Health. Vol. 6, No. 3, 2018 , pp. 82-85. doi: $10.11648 /$ j.sjph.20180603.13

Received: April 12, 2018; Accepted: May 8, 2018; Published: May 31, 2018

\begin{abstract}
Background: Placenta accreta is a general term used to describe the clinical condition when part of the placenta, or the entire placenta, invades and is inseparable from the uterine wall. Its incidence is growing due to the rising rate of cesarean sections and advanced maternal age on delivery. It is becoming the foremost cause of obstetric hemorrhage leading to significant maternal and fetal morbidity and even mortality. Rarely placenta accreta may lead to spontaneous uterine rupture in the second or third trimester. Case report: A 28-year-old woman gravida 4, para 3, was admitted at the maternity ward of Mendefera regional referral hospital, at a gestational age of 27 weeks due to vaginal bleeding. She had history of 3 time's caesarian section. At admission her vital sign was stable and her complete blood count was normal, ultrasound showed anterior placentation with partial placenta previa. She was given Dexamethasone $6 \mathrm{gm}$. IM twice daily for 2 days to enhance lung maturity. At 36 weeks of gestation, she experienced massive vaginal bleeding. A decision was made to perform emergency cesarean section. The possibility of morbidly adherent placenta was considered. Intra-operatively, the placenta was found with engorged blood vessels under the rectus fascia with ruptured uterus and there was adhesion of rectus sheath with part of the uterus. A transverse uterine incision was made at the upper border of the placental attachment to uterus to deliver the fetus. After successful delivery of the fetus, the placenta was found to be densely adhered to the lower uterine segment, penetrating through it and adhered to the posterior wall of the urinary bladder. It was decided to do caesarian hysterectomy with the placenta left in situ. During discharge both the mother and the baby were in good condition. Conclusion: Placenta accreta is a potentially life-threatening obstetric condition that requires a meticulous approach to management. If a multiparous woman with a previous caesarian section is found to have placenta previa, the possibility of placenta accreta should be considered in the diagnosis of the patient. Grayscale ultrasonography is sufficient for the diagnosis of placenta accreta. The recommended management of placenta accreta is planned caesarian hysterectomy.
\end{abstract}

Keywords: Placenta Previa, Caesarian Hysterectomy, Placenta Percreta, Outcome

\section{Introduction}

Placenta previa is the presence of placental tissue overlying or proximate to the internal cervical os. Its main complication is bleeding, which ranges from spotting to hemorrhagic $[1,2]$.
It is associated with severe maternal and fetal morbidity [3]. Its incidence is growing due to the rising rate of cesarean sections and advanced maternal age at delivery. The increasing incidence of cesarean delivery rate also leads to a marked increase in placenta accrete $[1,3]$. Placenta accreta is a 
general term used to describe the clinical condition when part of the placenta, or the entire placenta, invades and is inseparable from the uterine wall [4]. Both placenta previa and placenta accreta have the same major risk factors, namely tissue insult and scaring. The usual causes are previous cesarean section, myomectomy, hysteroscopicsurgery, suture of uterine perforation, infection and dilatation and curettage [5, $6,7]$. In the presence of placenta previa, the risk of having placenta accreta rises from $24 \%$ in women with one cesarean section to $67 \%$ for women who had a history of three or more previous cesarean sections [8, 9]. Placenta accreta is associated with major pregnancy complications, spontaneous uterine rupture including life-threatening maternal hemorrhage, large-volume of blood transfusion, and peripartum hysterectomy, causing a significant maternal and fetal morbidity and even mortality [3, 9-11].

There are limited data on its treatment and optimal management [12]. Majority of women with placenta accreta require hysterectomy. Although successful conservative management has been described, there are currently inadequate data to endorse this approach as routine management $[3,9,13]$.

\section{Case Report}

A 28-year-old woman (gravida 4, para 3) was admitted inMendefera Regional Referral Hospital, Maternity ward, at gestational age of 27 weeks due to vaginal bleeding. The bleeding was bright red and moderate in amount but sudden in onset. The mother had a history of 3 caesarian sections. On admission her vital signs, CBC (complete blood count) and hemoglobin $(14 \mathrm{gm} / \mathrm{dL})$ were normal, and her blood group was $\mathrm{O}^{+}$. Ultrasound examination showed normal fetal anatomy and growth, and confirmed the diagnosis of partial anterior placenta previa. The patient was informed about the diagnosis and its worst complications including the risk of preterm deliveries. She was given Dexamethasone $6 \mathrm{gm}$. intramuscular twice daily for 2 days to enhance lung maturity. Three units of blood were prepared for emergency operation. She was followed conservatively in the hospital for two months. During the follow-up period she had vaginal bleeding twice with moderate in amount. Her follow up ultrasound showed placenta previa with a loss of echo translucency behind the placenta.

At 36 weeks of gestation, she experienced massive vaginal bleeding and the possibility of morbidly adherent placenta was considered. A decision was made to perform emergency cesarean section. The patient and her family was informed all the possible complication, possible caesarian hysterectomies and its associated morbidity.

Under spinal anesthesia, the abdomen was opened in layer via lower midline incision. The placenta was found with engorged blood vessels under the fascia and there was adhesion of rectus sheath with part of the uterus. The uterus was ruptured and its upper and lower border was attached to the placenta (Figure 1). A transverse uterine incision was made at the upper border of the placental attachment to uterus.
A healthy female neonate weighing $2.5 \mathrm{~kg}$ with an Apgar score of $9 / 10$ at $1^{\text {st }}$ and $5^{\text {th }}$ minute was delivered. The placenta was found to be densely adhered to the lower uterine segment, penetrating through it and adhered to the posterior wall of the urinary bladder. An attempt was made to dissect the bladder from the placenta and lower uterine segment which ends up on injuring the bladder. The bladder was inspected for infiltration of the placenta and there was no visible infiltration on the posterior wall of the bladder. During the manipulation, there was profuse bleeding. It was decided to do caesarian hysterectomy with the placenta left in situ. The bladder was repaired in two layers after the hysterectomy. Blood loss was estimated around 1.5 liters and the patient was transfused 2 units of blood and 3 liters of crystalloid after the end of the operation. Parenteral antibiotic was continued for $24 \mathrm{hrs}$.

The post-operative course was uneventful. The urinary catheter was left in place for 10 days. The patient was discharged on the $11^{\text {th }}$ day of surgery voiding well. During her stays in the hospital there was no any complication, including urological problems. The neonate was active and feeding well during the hospital stay. The mother and the baby were discharged without any sequel.

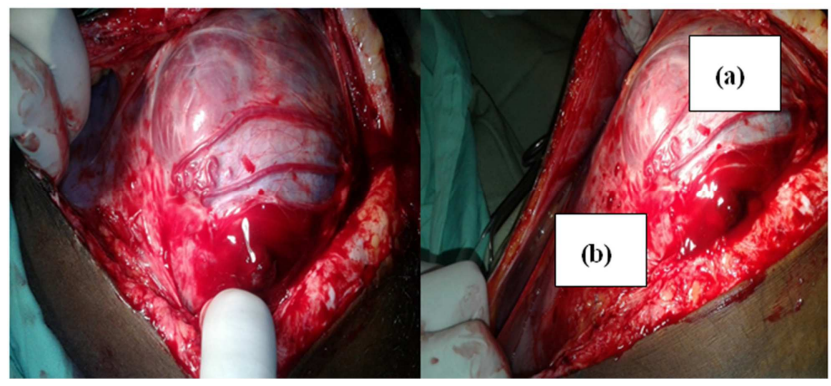

(a) ruptured uterus and (b) Lower segment of ruptured uterus

Figure 1. Placenta percreta and ruptured uterus.

\section{Discussion}

The incidence of placenta accrete has been reported to range 1 in 2,510 pregnancies in the1980s and 1990s as compared to 1 in 533 pregnancies by the year 2002, and placenta percreta, the rarest form of placenta accreta, comprised from $5 \%$ to $7 \%$ of all placenta accrete $[9,14]$. This rise in incidence rate is linked with persistent rise in caesarian section, uterine surgery, and increased parturient age and parity $[1-4,7,15]$. This condition has been present worldwide as the primary indication for emergency gravid hysterectomy which accounts for up to $50 \%$ of all emergency peri-partum hysterectomies [13]. In placenta percreta, rare variant of abnormal placentation with an incidence of 0.03 per 1,000 , placenta villi grow in to the myometrium, reaching or penetrating the uterine serosa, possibly extending beyond to the intra-abdominal or pelvic organs [14]. Placenta accreta may lead rarely to spontaneous uterine rupture in the second or third trimester which is consistent to the case presented [9].

This 28-year woman was presentedwith most significant risk factors for placenta accreta (placenta percreta), namely 3 prior caesarian sections, anterior placenta previa and parity. If 
a multiparous woman with a previous caesarian section is found to have placenta previa, the possibility of placenta accreta should be taken in to consideration during the diagnosis of the women, which is consistent to this case $[9,16$, 17]. There are studies that described the incidence of placenta accreta as directly linked with the increase in cesarean delivery. A large multicenter cohort study reported that the risk of placenta accreta on those women with placenta previa and prior caesarean as $3 \%, 11 \%, 40 \%, 61 \%$, and $67 \%$;for first, second, third, fourth, and fifth or more cesarean deliveries, respectively, [14].

Recent population based studies also documented that placenta accreta remains undiagnosed before delivery in half to two thirds of the cases [14]. However, having the above information, one should suspect placenta accreta and look for signs of accreta and evaluation of villous myometrial invasion on ultrasound examination in a mother who had history of caesarian section and mid-trimester placenta previa on ultrasound examination. Results from prospective cohort study conducted by Finberg et al., and Comstock et al., indicated that the sensitivity of gray-scale imaging alone in diagnosing placenta previa accreta in women with a prior caesarean delivery is $93 \%$ and $86 \%$, respectively $[18,19]$. In this case ultrasound examinations revealed anterior placenta previa with lose of echo translucencies that leadsto be conscious of placenta accrete and decision was made to perform emergency cesarean section. The maternal morbidity in women with placenta accreta is high, especially in cases of placenta percreta. There are studies that indicated mortality due to placenta accreta ranges from $7 \%$ to $22 \%[3,8]$. Hemorrhage and disseminated intravascular coagulation being the main cause of maternal death. In this cases intra-operative blood loses of the mother was estimated to be around 1.5 liters for this she was transfused 2 units of whole blood. Even though this amount of blood loss was very minimal as compared to some studies that documented, a mean blood lose in such a case during peri-partum hysterectomy as 2.5 liter [3]. As many as $90 \%$ of patients with placenta accreta require blood transfusion, and $40 \%$ require more than 10 units of packed red blood cells [4]. Bladder injury is one ofthe intra-operative complications that might happen during operation as it presented in this case.

Generally, the recommended management of suspected placenta accreta is planned preterm cesarean hysterectomy with the placenta left in situ because removal of the placenta is associated with significant hemorrhagic morbidity $[4,12,20$, 21]. However the treatment options vary from conservative approach to caesarian hysterectomy. There are studies that reported the overweighing advantage of conservative treatment for placenta accrete in helping the women and reducing morbidity by avoiding hysterectomy [22]. But in such a case for a delivering woman, who had a previous history of uterine rupture, three previous caesarian, parity and patient's obstetric history; the decision was emergency cesarean hysterectomy as a lifesaving procedure without considering conservative management.

\section{Conclusion}

In conclusion, placenta accreta is a potentially life-threatening obstetric condition that requires a meticulous approach to management. Multiparous women with a history of myometrial damage caused by caesarian delivery with anterior placenta previa overlying the uterine scar are at risk of placenta accreta. Recognizing these obstetrics risk factors allow the identification of most cases during the antepartum period. Diagnosing placenta accreta before delivery allows appropriate planning in attempt to improve the outcome of this increasingly more common major obstetrical complication. Gray-scale Ultrasonography is sensitive and specific enough for its definitive diagnosis. In general, all precaution and preparation should be done for emergency management of such cases, and planned caesarean hysterectomy with the placenta left in situ is the recommended management of placenta accreta because attempts at removal of the placenta are associated with significant hemorrhagic morbidity.

\section{Acknowledgements}

Dawit Sereke and Habte Hailemelekot have done surgery for the mother. Dawit Sereke, Abduselam Hagos and Elias Teages Adgoy designed the report, organized the information and wrote the report.

\section{Conflict of Interest Statement}

The authors declare that they have no conflict of interest.

\section{References}

[1] Miller DA, Chollet JA, Goodwin TM. Clinical risk factors for placenta previa-placenta accreta. American Journal of Obstetrics \& Gynecology. 1997 Jul 1; 177(1):210-4.

[2] Jameela S, Policarp B, Thankam U, Chellamma N. A Comparative Study on Maternal and Fetal Outcome in Cases of Placenta Previa with Previous Cesarean Section and Without Previous Cesarean Section.

[3] Sparić R, Mirković L, Ravilić U, Janjić T. Obstetric complications of placenta previa percreta. Vojnosanitetskipregled. 2014; 71 (12):1163-6.

[4] ACOG committee opinion. Placenta accreta. ACOG women's health care physicians, July 2012; (529).

[5] Wu S, Kocherginsky M, Hibbard JU. Abnormal placentation: twenty-year analysis. American Journal of Obstetrics \& Gynecology. 2005 May 1; 192 (5):1458-61.

[6] Matsubara S, Takahashi H, Lefor AK. The "morbidly adherent placenta" team: recognition and respect are needed. American Journal of Obstetrics \& Gynecology. 2017 Jul 1; 217 (1):101-2.

[7] Armstrong-Kempter S, Kapurubandara S, Trudinger B, Young N, Arrage N. A Case of Placenta Percreta Managed with Sequential Embolisation Procedures. Case Reports in Obstetrics and Gynecology, 2018. 
[8] Guleria K, Gupta B, Agarwal S, Suneja A, Vaid N, Jain S. Abnormally invasive placenta: changing trends in diagnosis and management. ActaobstetriciaetgynecologicaScandinavica. 2013 Apr 1; 92 (4): 461-4.

[9] Oyelese Y, Smulian JC. Placenta previa, placenta accreta, and vasa previa. Obstetrics \& Gynecology. 2006 Apr 1; 107 (4): 927-41.

[10] Biler A, Ekin A, Gezer C, Apaydın N, Solmaz U, Özeren M. Spontaneous uterine rupture due to placenta percreta in second trimester of pregnancy: A case report. Gazi Medical Journal. 2016 Jun 23; 27 (3).

[11] Farooq F, Siraj R, Raza S, Saif N. Spontaneous Uterine Rupture Due to Placenta Percreta in a 17-Week Twin Pregnancy. Journal of the College of Physicians and Surgeons--Pakistan: JCPSP. 2016 Nov; 26 (11): 121-3.

[12] Fitzpatrick KE, Sellers S, Spark P, Kurinczuk JJ, Brocklehurst P, Knight $\mathrm{M}$. The management and outcomes of placenta accreta, increta, and percreta in the UK: a population-based descriptive study. BJOG: An International Journal of Obstetrics \&Gynaecology. 2014 Jan 1; 121 (1): 62-71.

[13] Zelop CM, Harlow BL, FrigolettoJr FD, Safon LE, Saltzman DH. Emergency peripartum hysterectomy. American journal of obstetrics and gynecology. 1993 May 1; 168 (5): 1443-8.

[14] Chen C, Wang Y, Lin J, Chiu Y, Wu H, and Liu W. Journal of Obstetrics and Gynecology Research @2010 Japan Society of Obstetrics and Gynecology. 2010.

[15] Jauniaux E, Bhide A. Prenatal ultrasound diagnosis and outcome of placenta previa accreta after cesarean delivery: a systematic review and meta-analysis. American Journal of Obstetrics \& Gynecology. 2017 Jul 1; 217 (1): 27-36.
[16] David SN, Shibu CK, Mariam KT, Usha S, Supriya K, Bino B. Endovascular balloon occlusion of the aorta for placenta percreta during cesarean hysterectomy. Indian Journal of Case Reports. 2018; 4 (1): 14-6.

[17] Shetty S, Bagade P, Kumar S. CASE REPORT: UTERUS SAVED IN A CASE OF ANTENATALLY DIAGNOSED PLACENTA ACCRETA. GLOBAL JOURNAL FOR RESEARCH ANALYSIS. 2018 Feb 8; 6 (11).

[18] Finberg HJ, Williams JW. Placenta accreta: prospective sonographic diagnosis in patients with placenta previa and prior cesarean section. Journal of ultrasound in medicine. 1992 Jul 1; 11 (7): 333-43.

[19] Comstock CH, Love JJ, Bronsteen RA, Lee W, Vettraino IM, Huang RR, Lorenz RP. Sonographic detection of placenta accreta in the second and third trimesters of pregnancy. American Journal of Obstetrics \& Gynecology. 2004 Apr 1; 190 (4): 1135-40.

[20] Kayem G, Davy C, Goffinet F, Thomas C, Clement D, Cabrol D. Conservative versus extirpative management in cases of placenta accreta. Obstetrics \& Gynecology. 2004 Sep 1; 104 (3): 531-6.

[21] Mehdi ParvaMD, Dmitri Chamchad, Joan Keegan, Andrew Gerson, Jay Horrow. Placenta percreta with invasion of the bladder wall: management with a multi-disciplinary approach. A case report. Journal of Clinical Anesthesia (2010) 22, 209212.

[22] Sentilhes L, Ambroselli C, Kayem G, Provansal M, Fernandez H, Perrotin F, Winer N, Pierre F, Benachi A, Dreyfus M, Bauville E. Maternal outcome after conservative treatment of placenta accreta. Obstetrics \& Gynecology. 2010 Mar 1; 115 (3): 526-34 